\title{
Evaluation of drag and lift in the internal flow field of a dual rotor spinning unit via CFD
}

\author{
Nicholus Tayari Akankwasa ${ }^{1}$, Huiting Lin and Jun Wang ${ }^{1,2, a}$ \\ ${ }^{1}$ College of Textile, Donghua University, Shanghai, 201620, China \\ ${ }^{2}$ The Key Lab of Textile Science and Technology, Ministry of Education, Shanghai 201620, China
}

\begin{abstract}
In the present study, we evaluate the drag and lift magnitude in the new dual-feed rotor spinning unit using computational fluid dynamics technique. We adopt theoretical and numerical approach based on FLUENT to investigate the influence of new design on the drag and lift in the rotor interior. Results reveal that the drag and lift inside the rotor of the proposed model are reduced by $60-80 \%$ and $50-66 \%$ respectively as compared to the conventional unit. The velocity and pressure profiles become evenly distributed in the dualfeed rotor interior as opposed to the conventional rotor spinning unit and this modification is anticipated to improve fiber configurations. This phenomenon can be utilized to further optimize the rotor spinning unit and other wall-bounded engineering problems.
\end{abstract}

\section{Introduction}

In the fluid dynamics concept, previous studies have focused more on the turbulent viscosity, flocculation, eddies and vortices among other flow properties. Growing interest in drag reduction in flows has increased in the recent years. Principally, the drag and lift phenomenon has been widely applied in solving aerodynamics and aeronautics problems. For wall-bounded flows, it is important to note that drag and lift has a significant impact on the processing of fibers and polymers. Witold Brostow[1] reported a comprehensive review on drag reduction in flow covering the applications, mechanisms, and prediction. In his review, Witold Brostow[1] reported an increasing interest of application of drag reduction phenomenon in turbulent flows. Unlike the laminar flows, turbulent flows are rather chaotic, complex and associated with high Reynolds numbers. For effective evaluation of turbulent flows, drag and lift assessment can be helpful in benchmarking several engineering problems. Roberto Benzi[2] reported a well-articulated review on drag reduction by polymers in wall-bounded turbulence. The review reveals that the amount of polymer stretching transversely to mean flow plays a major role in understanding the phenomenon of drag reduction. Study on the behavior of drag and lift forces in the flows is a significant phenomenon that gives a comprehensive understanding of a given processes, which can help to improve the quality and handle costs efficiently.[3-5]

Numerical studies in textile processing have tremendously risen in the past decade with authors focusing on finite element methods to study fiber processing techniques more comprehensively[6-13]. In all industrial rotor spinning machines, the opening of the fibers is performed by the opening roller,

\footnotetext{
${ }^{a}$ Corresponding author: junwang@dhu.edu.cn
} 
in which sliver is fed from a single point. Increasing the number of feed rollers from one to two may improve fiber opening and trash ejection by two-step loading in the opening zone of the roller. Peyravi et al.[13] and Wang et al.[14] reported the dual-feed concepts that could spin yarns with better yarn properties. Their remarkable findings have significantly contributed to rotor spinning research with critical insights towards design modification, yarn quality improvement and reduction in production costs. Lin et al.[8] applied numerical methods to study rotor spinning process behavior, especially in the rotor interior. Their findings revealed a significant relationship between the geometrical characteristics, spinning parameters and airflow characteristics.

Our recent works on the new spinning dual-feed rotor spinning machine explored the 3D airflow domain with a focus on the effect of varying air velocity on the flow characteristics in the rotor.[12] Results showed that turbulence strength and Re increased with increase in inlet air velocity. Furthermore, pressure profiles and velocity distribution were affected as compared to the findings on the conventional rotor spinning unit by Lin et al.[8] Recent studies on drag and lift in turbulent flows have applauded the significance of the lift and drag in internal flows. This piece of work seeks to evaluate the drag and lift forces associated in the new dual-feed model. The rotor dimensions in this study were based on the model designed by Wang et al.[14] We explore the effects of the second channel on the drag and lift forces using numerical methods based on FLUENT. In the second section, we present a brief mathematical and numerical model. Section 3 provides an account of the airflow regime based on velocity and pressure contours and calculation of drag and lift. Finally, conclusions are drawn.

\section{Theoretical model and numerical procedure}

\subsection{Dual-feed rotor structure}

Figure 1 shows the geometrical model of the proposed dual-feed rotor spinning machine. The new design has two transfer channel inlets as opposed to the conventional unit[8]. The airflow is simulated from the two velocity inlets concurrently to the rotor interior up to the doffing tube. The Cartesian coordinate system is used. The origin of the model is situated at the center of the rotor, and one of the transfer channels lies in the positive $x-y$ plane, and the another one is in the negative $x-y$ plane. The rotor is region shares the three axes.

To demonstrate the effect of the dual-unit principle, we evaluate the drag and lift inside the rotor due to the effect of the second channel in the rotor.

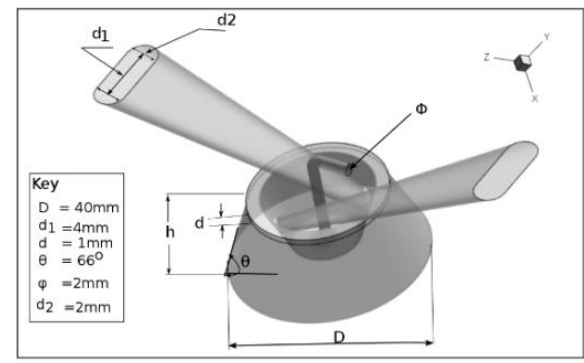

Figure 1. Geometrical profiles of a 3-D model of the dual rotor spinning unit.

\subsection{Governing equations}

Considering air flowing through the model domain, the flow direction originates from the channel inlets towards the rotor. The airflow in the rotor unit has Mach number of not more than 0.3 [5] so it can be regarded as incompressible. Reynolds number in the rotor spinning box is considerably high, up to 6000 and the flow is turbulent[7,8]. The equations adopted in our simulation intent hypothesize that the airflow in the rotor zone is viscous, incompressible and isentropic. The governing equations are articulate as follows; 


$$
\frac{\partial v}{\partial t}+\nabla \cdot \rho . \quad \vec{v}
$$

$\rho$ is the fluid density, $v$ is a velocity vector. Equation 1 implies that, the sum of the time-dependent change of density $\rho$ and the three-dimensional of the current density $\overrightarrow{\rho v}$ is zero.

$$
\frac{\partial\left(\rho u_{i}\right)}{\partial t}+\operatorname{div}\left(\rho \mathrm{u} u_{i}\right)=\operatorname{div}\left(\mu \operatorname{grad} u_{i}\right)-\frac{\partial p}{\partial x_{i}}+S_{i}
$$

The turbulent viscosity term $\mu_{t}$ expresses the relationship between the turbulent kinetic energy $\mathrm{k}$ and the turbulent dissipation rate $\varepsilon$ (see equation 3 ). The vortex viscosity term $\mu_{t}$ in the flow zone is expressed in terms of $\mathrm{k}$ and $\varepsilon$ (see equations 3-5).

$$
\begin{aligned}
& \mu_{t}=\rho C_{\mu} \frac{k^{2}}{\varepsilon} \\
& \frac{\partial(\rho k)}{\partial t}+\frac{\partial\left(\rho k u_{i}\right)}{\partial x_{j}}=\frac{\partial}{\partial x_{j}}\left[\left(\mu+\frac{\mu_{t}}{\sigma_{k}}\right) \frac{\partial k}{\partial x_{j}}\right]+G_{k}-\rho_{\varepsilon} \\
& \frac{\partial(\rho \varepsilon)}{\partial t}+\frac{\partial\left(\rho \varepsilon u_{i}\right)}{\partial x_{j}}=\frac{\partial}{\partial x_{j}}\left[\left(\mu+\frac{\mu_{t}}{\sigma_{\varepsilon}}\right) \frac{\partial k}{\partial x_{j}}\right]+\frac{C_{\left.\right|_{\varepsilon} \varepsilon}}{k} G_{k}-C_{2 \varepsilon} \rho \frac{\varepsilon^{2}}{k},
\end{aligned}
$$

The default values of constants $C_{\mu}, C_{\mathrm{I}_{\varepsilon}}, C_{2 \varepsilon}, \partial k$, and $\sigma_{\varepsilon}$ is same to literature[8].

\subsection{Numerical and solution procedure}

The investigation is performed using a three-dimensional numerical simulation based on the standard $\mathrm{k}-\varepsilon$ two-equation turbulence model. The Navier-Stokes equations are numerically integrated using the pressure-velocity coupling and SIMPLE algorithm scheme. In this numerical simulation procedure, the standard pressure spatial discretization is considered, the momentum, kinetic energy, and turbulent dissipation rate are evaluated by the second order upwind method to improve the accuracy of the model. The solution is initialized by hybrid methods and the convergence criterion of volume and surface monitors assigned to $10^{-6}$. Due to incompressible effects, the pressure-velocity approach adopted works on the principle that performs simultaneous solutions of the conservation of mass, energy and turbulent kinetic energy as well as its dissipation rate equations within the physical domain. We used ANSYS ICEM CFD, release 14.5 to develop, design, mesh and simulate our model. Figure 2 shows the mesh topology and boundary conditions. The parameters considered in this model are similar to our recent works on the 3-D airflow field[12]. Mesh independence tests were performed prior final simulation runs. Details of grid independence tests for the reported model are well explained in Akankwasa et al.[12]

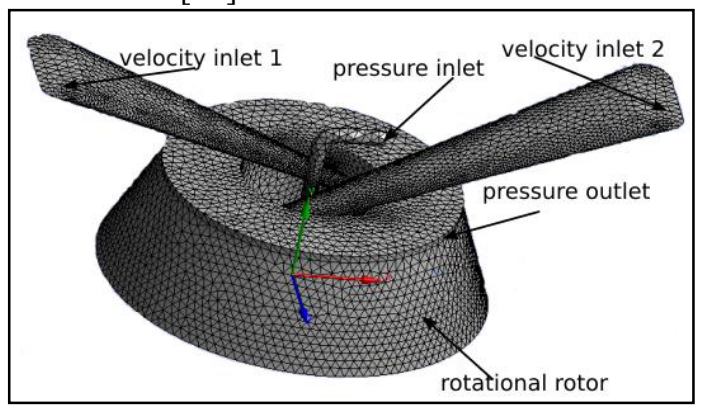

Figure 2. Mesh topology and boundary conditions of the dual-feed rotor spinning unit.

\subsection{Drag and lift calculation}

The drag force experienced by a flexible fiber acts in the direction of the flow velocity and is characterized by the drag coefficient. In general, the drag coefficient can be defined by equation 6 .

$$
C_{D}=\frac{F_{D}}{\frac{1}{2} \rho u^{2} A_{f}}
$$


Where $F_{D}$ is the actual drag force, $\mathrm{u}=u_{\infty}-U_{f}$ is the velocity of the fiber to the local undisturbed fluid velocity $u_{\infty}, \rho$ is the fluid density, and $A_{p}=\frac{\pi}{4} d_{f}$ the reference area with $d_{p}$ being the fiber diameter. To compute the total drag force, equation 7 is considered;

$$
F_{D}=C_{f} \pi d_{f}(v-u) l
$$

Where $C_{f}$ is the coefficient of friction between the fiber and air and a given fiber in motion, $\rho$ is the air density, $d_{f}$ is the fiber diameter, $\mathrm{v}$ is the air velocity, $\mathrm{u}$ is the fiber velocity and $l$ is the fiber length. The numerical simulation data provides the values of parameters such as air density, air velocity through probing specific points in the model.

In the drag equation, $\pi$ and $\mathrm{d}$ are theoretically constant, however $C_{f}$ varies with the relative velocity between air and the fiber. The influence of the coefficient of friction was treated as minimal in this evaluation procedure as proposed by previous studies.[10,11] To simplify the evaluation, we propose treating $C_{f}, \pi$, and $\mathrm{d}$ as 1 , such that the drag force in the rotor can be evaluated as equation 8 below.

$$
F_{D}=\rho(v-u) d l
$$

Considering an element (see Figure 3 ) inside the rotor with two connecting points, $a$ and $b$, where $a$ is the is characterized by velocity $v_{a}$ and density $\rho_{a}$, and point $\mathrm{b}$ presented with different velocity and density as shown in Figure 3.

Figure 3: Representation of fiber the rotor

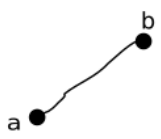

Physical parameters of fiber element such as $\rho$ and $v$ in Figure 3 can be disposed as;

$$
\rho=\frac{1}{2}\left(\rho_{a}+\rho_{b}\right), \quad \mathrm{v}=\frac{1}{2}\left(v_{a}+v_{b}\right)
$$

The total drag is calculated by summation of drag force per unit length (see equation 10).

$$
\mathrm{F}=\sum F_{D} / L
$$

Lift force on a particle not aligned with the flow velocity is a result of the non-axisymmetric flow field. It acts in the direction perpendicular to the area of velocity its coefficient can be expressed as equation 11.

$$
C_{L}=\frac{F_{L}}{\frac{1}{2} \rho u^{2} A_{f}}
$$

$F_{L}$ is the exact lift force acting orthogonal to the flow velocity of the fluid. Based on the theoretical modelling and simulation data, we integrate the total drag and lift in the conventional and rotor spinning unit and analysed the results.

\section{Results and discussions}

\subsection{Evaluation of the airflow regime}

To visualize the effect of the second dual channel on velocity profiles, we extracted the flow domain at $\mathrm{z}=0.000384 \mathrm{~m}$ perpendicular to the $\mathrm{x}-\mathrm{y}$ plane. Figure 4 (a) shows the dual-feed rotor spinning unit and (b) shows the conventional rotor unit. As seen in Figure 4, the velocity distribution in the transfer channels exhibits similar patterns in the two models. We notice a difference inside the rotor at the positions near the transfer channel exits. The air velocity of $40-60 \mathrm{~m} / \mathrm{s}$ is observed, and the new dualfeed model reveals a much even distribution compared to the conventional unit. The $3 \mathrm{D}$ airflow regime in the dual-feed model has been extensively simulated and verified in our previous works[12]. This section is entirely dedicate on drag and lift evaluation in the proposed dual-feed rotor spinning concept compared to the traditional open-end spinning system(rotor spinning). 


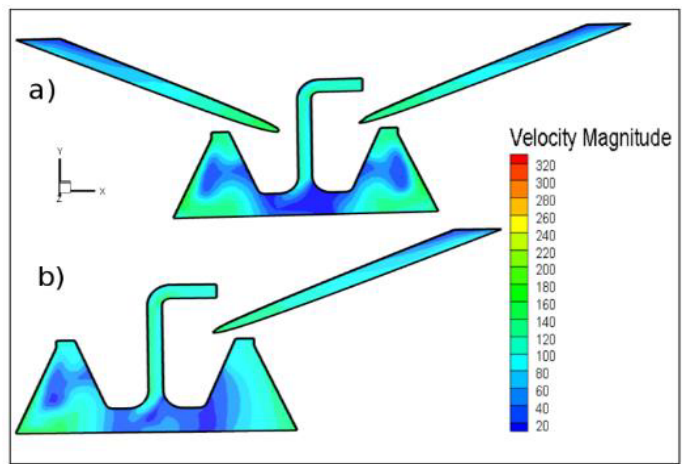

Figure 4. Velocity magnitude comparison: (a) modified rotor spinning unit, (b) conventional rotor spinning unit.

The overall velocity magnitude observed, shows that the second feed introduced changes the airflow dynamics. Our results quantitatively agree with previous studies by Kong and Platfoot[7], Lin et al.[8], and Lawrence and Chen[15,16]. The velocity profiles in the dual-feed model predict a significant shift in the way fibers can be aligned inside the rotor. Since both sides, the rotor interior is characterized by balance velocity flow. Our simulation results anticipate improved fiber alignment however, this need to be further proved experimentally.

Figure 5 shows the pressure distribution in the rotor of the conventional and modified rotor spinning process. The region opposite the transfer channel outlet of the conventional model is characterized by pressure ranging from -8000 to -6000 pa with the center largely dominated by $8000 \mathrm{pa}$. On the centrally, the pressure distribution in the modified model is evenly distributed with the transfer channel exit positions occupied by traces of positive pressure of up to 6000pa.
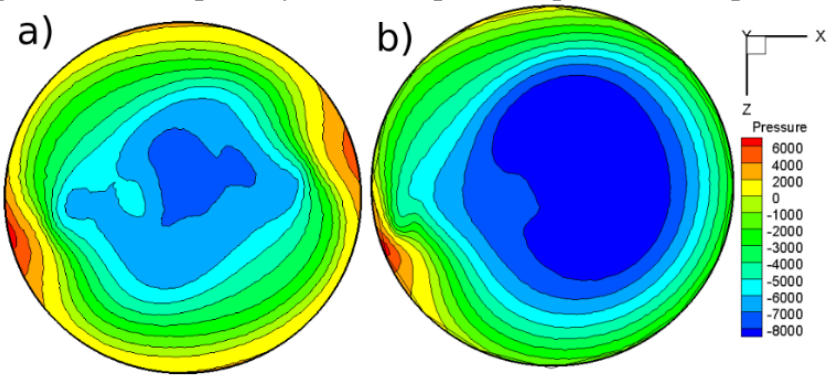

Figure 5. Pressure profiles: (a) dual-feed rotor spinning unit (b) conventional rotor spinning system

The pressure difference in internal flow system is a very crucial parameter because it influences the forces and moments such as drag and lift forces. The effect generated by pressure difference eventually influences the airflow dynamics and hence the production process.[11] Even pressure distribution achieved through additional transfer channel will affect the fiber processing mechanics. This observation agrees with Peyravi et al.[13] Findings when they improvised a dual-feed unit to study the yarn characteristics.

\subsection{Evaluation of drag and lift}

Lift in fluid flow is brought about by the pressure difference that exists in the flow regime. In our study, we integrate drag and lift in the two models to evaluate the difference between the models. Figure 6 (a) shows the lift force generated in the conventional unit and the dual-feed with a change in the outlet pressure. Results demonstrate that with the new model reduces the drag force by 50 to $60 \%$. Previous analysis revealed a huge reduction in the drag force as well. Therefore, the new model is anticipated to have a greater positive influence on the yarn quality properties. 


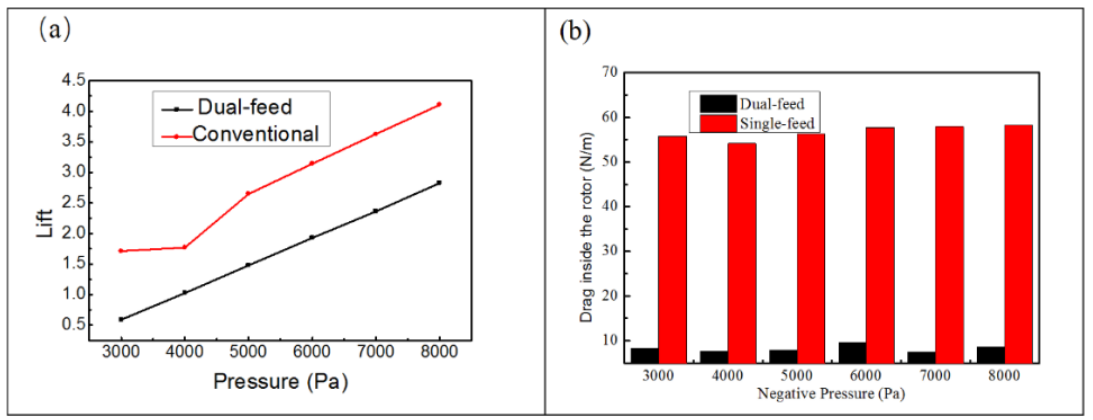

Figure 6. (a) lift evaluation, (b) drag evaluation.

Drag is the resistance to flow which acts opposite to the relative motion of any object moving in a fluid or air. Drag exists either between two layers or surfaces or a fluid and a solid surface. In this simulation intent, we evaluated the design of drag that exists in the rotor interior. Even if the fibers are flexible and the rotor walls smooth, for evaluation of physics of flows, form, and frictional drag exists during fiber processing into yarn. Evaluation of the two simulated models revealed drag reduction ranging from 60 to $80 \%$ due to the existence of the second feeding unit (see figure 6 (b)).

\section{Conclusions}

A standard k-epsilon model is implemented to evaluate the impact of introducing a second transfer channel in the rotor spinning unit on the drag and lift forces. The flow field is assessed based on the simulation data. Based on results, we conclude that the second channel imparts a significant effect on the flow behavior. The air velocity in the rotor interior of the new model becomes evenly distributed compared to the conventional one. The drag and lift force shows $60-80 \%$ and $50-66 \%$ reduction respectively. The new model can improve the flow stability in the rotor interior and could improve the fiber alignment and configuration.

\section{Acknowledgement}

This work was supported by the Key Grant Project of the Ministry of Education of the People's Republic of China (Grant No. 113027A)

\section{References}

1. W. Brostow, J. Ind. Eng. Chem. 14, 409 (2008).

2. R. Benzi, Phys. D Nonlinear Phenom. 239, 1338 (2010).

3. K. Iwamoto, K. Fukagata, N. Kasagi, and Y. Suzuki, Phys. Fluids 17, (2005).

4. S. Lardeau and M.A. Leschziner, Phys. Fluids 25, (2013).

5. S. Tamano and M. Itoh, J. Turbul. 13, N9 (2012).

6. C.T. DeGroot, C. Wang, and J.M. Floryan, J. Fluids Eng. 138, 1 (2016).

7. L.X. Kong and R.A. Platfoot, Text. Res. J. 67, 269 (1997).

8. H.T. Lin, Y.C. Zeng, and J. Wang, Text. Res. J. 86, 115 (2016).

9. V.C. PATEL, W. RODI, and G. SCHEUERER, AIAA J. 23, 1308 (1985).

10. Y.-S. Wang, W.-X. Huang, and C.-X. Xu, Fluid Dyn. Res. 48, 55501 (2016).

11. M. Xiao, H.-S. Dou, and C. Wu, Text. Res. J. (2016).

12. N.T. Akankwasa, H. Lin, Y. Zhang, and J. Wang, Text. Res. J. (2016).

13. A. Peyravi, S. Eskandarnejad, and M.B. Moghadam, J. Text. Inst. 105, 377 (2014).

14. W. Jun, J.X.Yan, Y.Liang, Z.Yuze, G.Wei, and F.Ting, CN103911696A (2014).

15. C.A. Lawrence and K.Z. Chen, J. Text. Inst. 77, 201 (1986).

16. C.A. Lawrence and K.Z. Chen, J. Text. Inst. 77, 139 (1986). 